South Gondar Trachoma Impact Evaluation Survey - Amhara, Ethiopia: 2011

\title{
HOUSEHOLD QUESTIONNAIRE
}

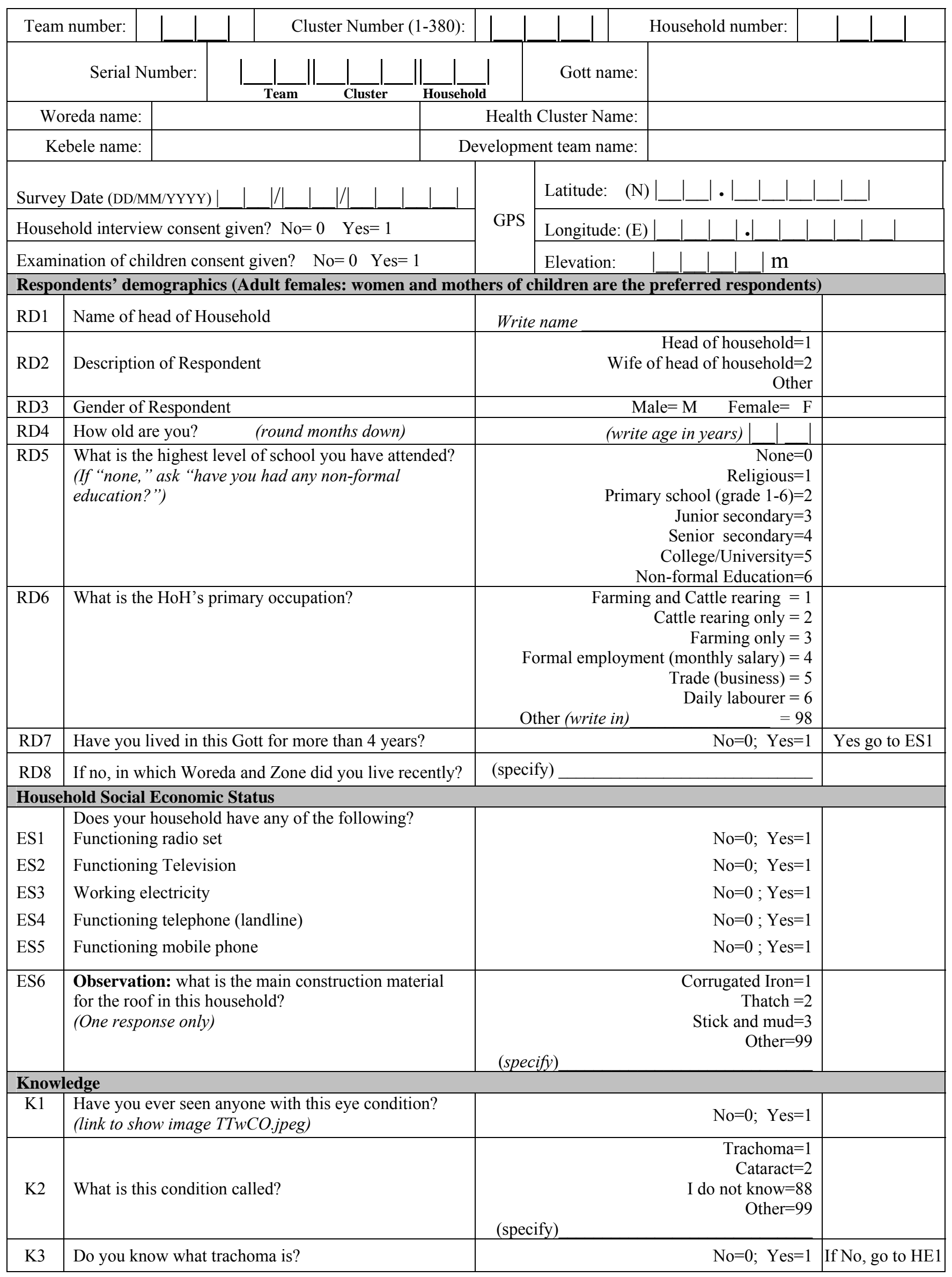




\begin{tabular}{|c|c|c|c|}
\hline K4 & $\begin{array}{l}\text { In the end, what can happen to a person who has } \\
\text { trachoma? (multiple response) } \\
\text { (After each response ask 'anything else?' Do not read } \\
\text { choices. Please mark all responses given.) }\end{array}$ & $\begin{array}{llr} & \text { K4.1 } & \text { Nothing happens } \square \\
& \text { K4.2 } & \text { Blindness } \square \\
& \text { K4.3 } & \text { Reduced vision } \square \\
& \text { K4.88 } & \text { I do not know } \square \\
& \text { K4.99 } & \text { Other } \square \\
\text { (specify) } & & \\
\end{array}$ & \\
\hline K5 & $\begin{array}{l}\text { How can someone protect him/herself from } \\
\text { trachoma/trichiasis? } \\
\qquad \text { (multiple response) } \\
\text { (After each response ask 'anything else?' Do not read } \\
\text { choices. Please mark all responses given.) }\end{array}$ & $\begin{array}{rr}\text { K5.1 } & \text { Face washing/hygiene } \square \\
\text { K5.2 } & \text { Take antibiotics or medicine } \square \\
\text { K5.3 } & \text { Trichiasis surgery } \square \\
\text { K5.4 } & \text { Keeping environment clean } \square \\
\text { K5.5 } & \text { Using pit latrines } \square \\
\text { K5.88 } & \text { I do not know } \square \\
\text { K5.99 } & \text { Other } \square \\
\text { (specify) }\end{array}$ & \\
\hline Heal & Education & & \\
\hline HE1 & Have you ever heard health information on trachoma? & Yes $=1$ & If No go to WS1 \\
\hline HE2 & $\begin{array}{l}\text { Where did you hear the trachoma information? } \\
\qquad \text { (multiple response) } \\
\text { (After each response ask 'anything else?' Do not read } \\
\text { choices. Please mark all responses given.) }\end{array}$ & $\begin{array}{cr}\text { HE2.1 } & \text { Trachoma volunteers } \square \\
\text { HE2.2 } & \text { Health extension worker } \square \\
\text { HE2.3 } & \text { Mass media (TV, radio, etc) } \square \\
\text { HE2.4 } & \text { Health facility } \square \\
\text { HE2.5 } & \text { Community gatherings } \square \\
\text { HE2.6 } & \text { School } \square \\
\text { HE2.7 } & \text { School child } \square \\
\text { HE2.99 } & \text { Other } \square \\
\text { (specify) } & \end{array}$ & \\
\hline HE3 & $\begin{array}{l}\text { What information about trachoma did you hear? } \\
\qquad \text { (multiple response) } \\
\text { (After each response ask 'anything else?' Do not read } \\
\text { choices. Please mark all responses given.) }\end{array}$ & $\begin{array}{cr}\text { HE3.1 } & \text { Causes of trachoma } \square \\
\text { HE3.2 } & \text { Transmission of trachoma } \square \\
\text { HE3.3 Latrine construction and use } \square \\
\text { HE3.4 } \\
\text { HE3.5 } & \text { Face washing } \square \\
\text { HE3.6 } & \text { Antibiotics treatment } \square \\
\text { HE3.99 } & \text { Trichiasis surgery } \square \\
\text { (specify) } & \text { Other } \square \\
\end{array}$ & \\
\hline \multicolumn{4}{|c|}{ Water source and access (Ask person responsible for water collection) } \\
\hline WS1 & $\begin{array}{l}\text { What is the main source of water your } \\
\text { household uses for bathing? }\end{array}$ & $\begin{array}{r}\text { Unprotected spring }=1 \\
\text { Protected spring }=2 \\
\text { Unprotected dug well }=3 \\
\text { Hand pump/Tube well / borehole }=4 \\
\text { Surface water (river, dam, lake, stream, canal) }=5 \\
\text { Public piped water/ tap/standpipe }=6 \\
\text { Private piped into Yard } / \text { dwelling }=7 \\
\text { Rainwater collection }=8 \\
\text { Other (specify)_ }=99 \\
\end{array}$ & \\
\hline WS2 & $\begin{array}{l}\text { What is the main source of water your } \\
\text { household uses for washing clothes? }\end{array}$ & $\begin{array}{r}\text { Unprotected spring }=1 \\
\text { Protected spring }=2 \\
\text { Unprotected dug well }=3 \\
\text { Hand pump/Tube well / borehole }=4 \\
\text { Surface water (river, dam, lake, stream, canal })=5 \\
\text { Public piped water/ tap/standpipe }=6 \\
\text { Private piped into Yard/dwelling }=7 \\
\text { Rainwater collection }=8 \\
\text { Other (specify) }\end{array}$ & \\
\hline
\end{tabular}




\begin{tabular}{|c|c|c|c|}
\hline WS3 & $\begin{array}{l}\text { What is the main source of water your } \\
\text { household uses for drinking? }\end{array}$ & $\begin{aligned} \text { Unprotected spring } & =1 \\
\text { Protected spring } & =2 \\
\text { Unprotected dug well } & =3 \\
\text { Hand pump/Tube well } / \text { borehole } & =4 \\
\text { Surface water (river, dam, lake, stream, canal) } & =5 \\
\text { Public piped water/ tap } / \text { standpipe } & =6 \\
\text { Private piped into Yard } / \text { dwelling } & =7 \\
\text { Rainwater collection } & =8 \\
& =99\end{aligned}$ & \\
\hline WS4 & $\begin{array}{l}\text { How long does a round trip take to collect } \\
\text { water from the source of water used for } \\
\text { bathing? }\end{array}$ & $\begin{aligned}<30 \text { minutes } & =1 \\
30 \text { minutes to } 1 \text { hour } & =2 \\
>1 \text { hour } & =3\end{aligned}$ & \\
\hline \multicolumn{4}{|c|}{ Face washing (Ask mothers or care givers of children) } \\
\hline FW1 & Do you have children under 5 years of age? & $\mathrm{No}=0 ; \quad \mathrm{Yes}=1$ & $\begin{array}{l}\text { If No, go to } \\
\text { PL1 }\end{array}$ \\
\hline FW2 & $\begin{array}{l}\text { How often are these children bathed? } \\
\text { (Indicate one answer) }\end{array}$ & $\begin{array}{r}\text { Never }=0 \\
\text { Every other day }=1 \\
\text { Once a day }=2 \\
\text { Twice a day }=3 \\
\text { Three or more times a day }=4 \\
\text { Other } \\
=99\end{array}$ & \\
\hline FW3 & $\begin{array}{l}\text { How often are the faces of children washed? } \\
\text { (Indicate one answer) }\end{array}$ & $\begin{array}{r}\text { Never }=0 \\
\text { Every other day }=1 \\
\text { Once a day }=2 \\
\text { Twice a day }=3 \\
\text { Three or more times a day }=4 \\
\text { Other } \\
=99\end{array}$ & \\
\hline \multicolumn{4}{|c|}{ Pit Latrines } \\
\hline $\begin{array}{l}\text { PL1 } \\
\text { PL2 } \\
\text { PL3 } \\
\text { PL4 }\end{array}$ & $\begin{array}{l}\text { Have you ever had a latrine in this household? } \\
\text { Currently, is there a latrine in this household (observed)? } \\
\text { Is this the first latrine in this household? } \\
\text { How long ago was the most recent latrine built? }\end{array}$ & $\begin{array}{r}\mathrm{No}=0 ; \mathrm{Yes}=1 \\
\mathrm{No}=0 ; \mathrm{Yes}=1 \\
\mathrm{No}=0 ; \mathrm{Yes}=1 \\
\text { MONTHS ago }\end{array}$ & $\begin{array}{l}\text { If No go to } \\
\text { LO2 (note there } \\
\text { may still be a } \\
\text { hand-washing } \\
\text { container in the } \\
\text { HH) }\end{array}$ \\
\hline \multicolumn{4}{|c|}{ Latrine observations } \\
\hline LO1 & Evidence of latrine usage (faeces in pit)? & $\mathrm{No}=0 ; \mathrm{Yes}=1$ & \multirow{4}{*}{$\begin{array}{l}\text { If no to LO2, } \\
\text { END }\end{array}$} \\
\hline LO2 & Hand washing container present? & $\mathrm{No}=0 ; \mathrm{Yes}=1$ & \\
\hline LO3 & Water in hand washing container? & $\mathrm{No}=0 ; \mathrm{Yes}=1$ & \\
\hline LO4 & Location of hand washing container? & At household $=2$ & \\
\hline
\end{tabular}


Serial Number

(same as HH quest.)

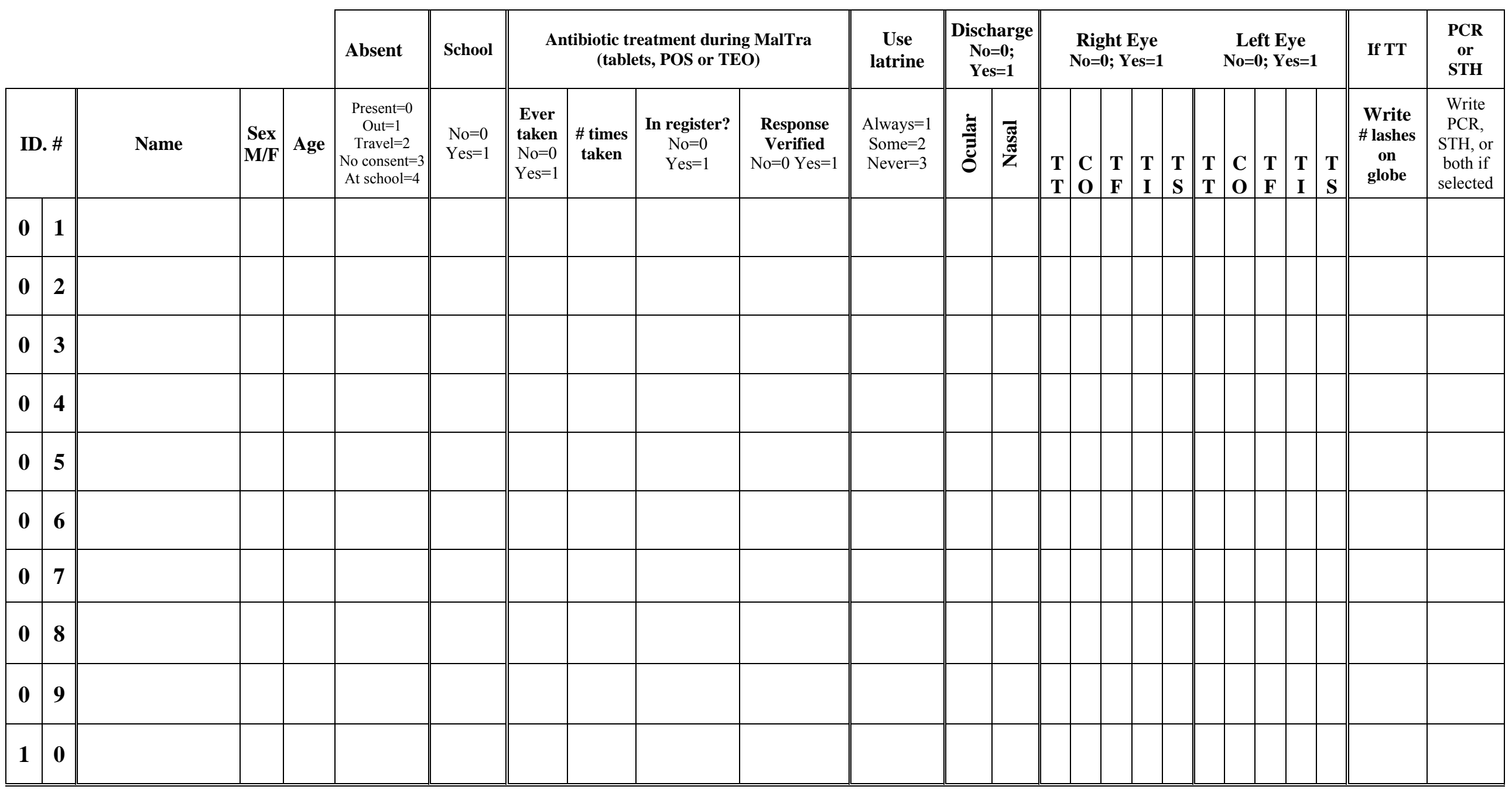

